\title{
Microstructure and energy dispersive diffraction reconstruction of 3D patterns of crystallographic texture in a shark centrum
}

\author{
Stuart R. Stock $\odot,{ }^{\text {a, } * \text { Paul E. Morse } \odot, ~}{ }^{\text {b,c }}$ Michala K. Stock, ${ }^{d}$ \\ Kelsey C. James $\odot{ }^{\text {e }}$ Lisa J. Natanson, ${ }^{\text {f Haiyan Chen, }}{ }^{\mathrm{g}}$ Pavel D. Shevchenko, \\ Evan R. Maxey ${ }^{\text {h }},{ }$ Olga A. Antipova, ${ }^{\text {h }}$ and Jun-Sang Park ${ }^{h}$ \\ ${ }^{a}$ Northwestern University, Simpson Querrey Institute, Feinberg School of Medicine, \\ Chicago Department of Cell and Developmental Biology, Illinois, United States \\ ${ }^{\mathrm{b}}$ Duke University, Department of Evolutionary Anthropology, Durham, North Carolina, \\ United States \\ ${ }^{\mathrm{c}}$ University of Florida, Florida Museum of Natural History, Gainesville, Florida, United States \\ ${ }^{\mathrm{d}}$ Metropolitan State University of Denver, Department of Sociology and Anthropology, \\ Denver, Colorado, United States \\ ${ }^{\text {e}}$ Southwest Fisheries Science Center, National Marine Fisheries Service, NOAA, La Jolla, \\ California, United States \\ ${ }^{\mathrm{f}}$ National Marine Fisheries Service, NOAA, (retired) Northeast Fisheries Science Center, \\ Narragansett, Rhode Island, United States \\ ${ }^{\mathrm{g}}$ Stony Brook University, Mineral Physics Institute, Stony Brook, New York, United States \\ ${ }^{\mathrm{h}}$ Argonne National Laboratory, Advanced Photon Source, Lemont, Illinois, United States
}

\begin{abstract}
Purpose: Tomography using diffracted $\mathrm{x}$-rays produces reconstructions mapping quantities such as crystal lattice parameter(s), crystallite size, and crystallographic texture, information quite different from that obtained with absorption or phase contrast. Diffraction tomography is used to map an entire blue shark centrum with its double cone structure (corpora calcerea) and intermedialia (four wedges).
\end{abstract}

Approach: Energy dispersive diffraction (EDD) and polychromatic synchrotron x-radiation at 6-BM-B, the Advanced Photon Source, were used. Different, properly oriented Bragg planes diffract different $\mathrm{x}$-ray energies; these intensities are measured by one of ten energy-sensitive detectors. A pencil beam defines the irradiated volume, and a collimator before each energysensitive detector selects which portion of the irradiated column is sampled at any one time. Translating the specimen along $X, Y$, and $Z$ axes produces a 3D map.

Results: We report 3D maps of the integrated intensity of several bioapatite reflections from the mineralized cartilage centrum of a blue shark. The $c$ axis reflection's integrated intensities and those of a reflection with no $c$ axis component reveal that the cone wall's bioapatite is oriented with its $c$ axes lateral, i.e., perpendicular to the backbone's axis, and that the wedges' bioapatite is oriented with its $c$ axes axial. Absorption microcomputed tomography (laboratory and synchrotron) and x-ray excited x-ray fluorescence maps provide higher resolution views.

Conclusion: The bioapatite in the cone walls and wedges is oriented to resist lateral and axial deflections, respectively. Mineralized tissue samples can be mapped in 3D with EDD tomography and subsequently studied by destructive methods.

(C) 2022 Society of Photo-Optical Instrumentation Engineers (SPIE) [DOI: 10.1117/1.JMI.9.3.031504]

Keywords: energy dispersive diffraction; microcomputed tomography; $\mathrm{x}$-ray fluorescence; shark; vertebra; mineralized cartilage; bioapatite.

Paper 21265SSR received Oct. 2, 2021; accepted for publication Jan. 6, 2022; published online Feb. 2, 2022.

*Address all correspondence to Stuart R. Stock, s-stock@northwestern.edu 

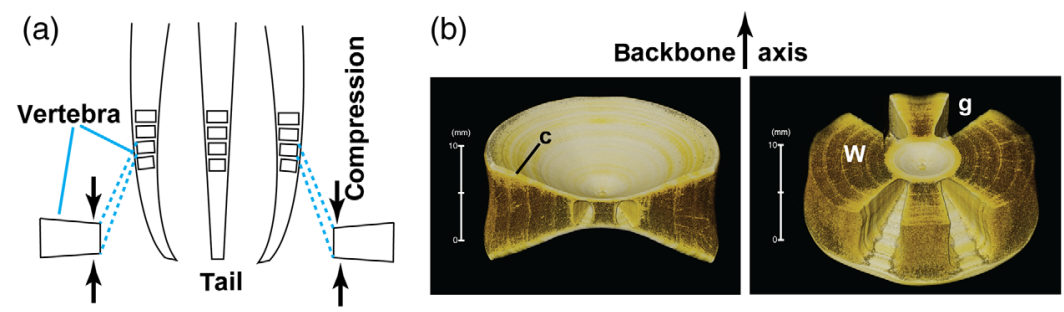

Fig. 1 (a) Vertebral compression when a shark swims, illustrated schematically by three positions of the tail. When the tail swings to the right (left tail diagram), the right side of the vertebra is compressed as indicated by the compression of the right side of the vertebra (left trapezoid with arrows indicating compression). As the right side of the schematic indicates, the vertebra's opposite (left) side compresses when the tail moves to the left. (b) Lab microCT-derived renderings of the blue shark centrum with the portions closest to the viewer rendered transparent (left, axial clipping plane; right, transverse clipping plane). The axial clipping plane shows the section orientation for x-ray fluorescence mapping (Fig. 4). Labels: c, hourglass-shaped cone of mineralized cartilage; $\mathrm{W}$, wedges of the intermedialia; $\mathrm{g}$, gaps separating the wedges.

\section{Introduction}

Sharks have cartilaginous skeletons, and each vertebra of their vertebral column contains a mineralized centrum that carries the loads generated during swimming. ${ }^{1}$ The structure and mineralization of the centra enable shark vertebrae to survive enormous compressive strains of $3 \%$ to $8 \%{ }^{2}$ for millions of cycles of loading, ${ }^{3}$ despite the absence of a repair mechanism analogous to remodeling in bone. Diffraction has shown that the centra's mineral is a bioapatite closely related to hydroxyapatite (hAp), ${ }^{4,5}$ and is organized similarly to the bone. ${ }^{6}$

Shark centra have complex 3D structures, and this paper describes the abdominal centra of the blue shark (Prionace glauca, Carcharhiniformes), the centra which experience very high, swimming-induced strains. Each centrum consists of an hourglass-shaped double cone, the corporus calcerea, of mineralized cartilage ("c" in Fig. 1). A fluid-filled inter-vertebral capsule lies between the cone walls of adjacent vertebrae. Within a blue shark centrum, the rostral and caudal cone walls are supported by the intermedialia consisting of four thick wedges ("W" in Fig. 1) with unmineralized gaps "g" separating the wedges. Centra macrostructure differs between carcharhiniform species. ${ }^{7}$ During swimming, both the left and right sides of shark abdominal centra experience an alternating pattern of compression and tension (Fig. 1). Some studies of the macroscopic strains in the shark spine have appeared, ${ }^{1,2,8}$ but little is known about the 3D distributions of displacement or strain within the complex structure of a shark centrum or even the presence or absence of bioapatite crystallographic texture.

The study reported below uses a novel approach, energy dispersive diffraction (EDD) with polychromatic synchrotron x-radiation, to map the 3D spatial organization of bioapatite and its crystallographic texture in a blue shark centrum. The novelty of this study stems from four factors. First, studies employing 3D diffraction mapping of mineralized tissue are relatively sparse. Second, engineering materials are infrequently studied with EDD and even less frequently with position resolution. Third, the authors are only aware of one paper applying EDD to a mineralized tissue sample. 9 Fourth, beyond the papers cited above, there are no diffraction studies of shark centra tissue.

In the present study, the authors ask whether weak bioapatite diffraction peaks can still produce an informative EDD reconstruction, whether these data reveal bioapatite crystallographic texture, and how the results relate to anatomical function and patterns of in vivo loading of the centrum. Results from microcomputed tomography (microCT) and from scanning x-ray excited X-ray fluorescence are used to help interpret the EDD maps. Prospects for future EDD mapping of shark centra are discussed as are ways in which similar data may be obtained for mineralized tissues of clinical interest.

\section{Materials and Methods}

One complete abdominal vertebra of a blue shark (Prionace glauca, Carcharhiniformes, from vertebrae numbers 81-84) was studied first with lab microCT and later with EDD mapping. 
The diameter and height of the vertebra's centrum were 24.3 and $12.7 \mathrm{~mm}$, respectively. A second vertebrae from the shark was cut into sections parallel to the vertebral column's axis and $<1$-mm thick (Fig. 1); these were used for fluorescence mapping. Similarly oriented blocks with roughly square cross-sections $\sim 2.5 \mathrm{~mm}$ across were cut for synchrotron microCT; at present, the investigators have not been able to cut $1 \times 1 \mathrm{~mm}$ cross-section blocks without gross distortion and they did not want to embed in plastic at this stage of the investigation.

The lab microCT scan of the frozen blue shark centrum was performed with the Nikon XTH 225 ST system at the Shared Materials Instrumentation Facility at Duke University. The centrum was placed approximately at the center of the scanner rotation axis. The x-ray tube was operated at $140 \mathrm{kVp}$ and $111 \mu \mathrm{A}$ with a $0.125-\mathrm{mm}$ thick $\mathrm{Cu}$ filter. Four frames were averaged per projection with $500 \mathrm{~ms}$ integration per frame, and 2500 projections were recorded over $360 \mathrm{deg}$. The volume was reconstructed with isotropic $18.4-\mu \mathrm{m}$ volume elements (voxels).

$\mathrm{X}$-ray excited $\mathrm{x}$-ray fluorescence mapping provided maps of elemental composition of a section of a blue shark centrum and was performed at two beam lines at the Advanced Photon Source (APS). The section was parallel to the clipping plane of the left image of Fig. 1(b). At beamline 8-BM-B, Kirkpatrick-Baez optics focused the beam of $15.3 \mathrm{keV}$ x-rays to $\sim 20-\mu$ m diameter. ${ }^{10}$ The sample was rastered across the beam, and maps of the fluorescent intensity were recorded. At beamline 2-ID-E, APS, Fresnel optics produced a 0.4 to $0.45 \mu$ m diameter beam of $13.7-\mathrm{keV}$ photons. A small portion of the same section was scanned at a much higher resolution than at 8-BM. The 2-ID-E apparatus is described elsewhere. ${ }^{11}$

Synchrotron microCT was performed at beamline 2-BM, APS. Multiple blocks cut from a blue shark centrum were imaged with $24.35 \mathrm{keV}$ x-ray photons and 1500 projections over $180 \mathrm{deg}$. The $2448 \times 2448$ voxel slices were reconstructed with $0.69-\mu \mathrm{m}$ voxels, covering a field of view (FOV) of diameter $\sim 1.7 \mathrm{~mm}$. The result was local tomographic imaging of the $\sim 2.5 \times 2.5 \mathrm{~mm}$ cross-sectional blocks. Local tomography ${ }^{12}$ was the compromise between the large number of FOV that were required and adequate spatial resolution to see the micrometer-level structure of the cone walls versus intermedialia. Specifically, the entire rostral to caudal length of each block needed to be covered (an average of $10 \mathrm{FOV}$ along this axis), and multiple blocks from multiple species were to be covered to build up a representative picture of each species. Precise measurement of linear attenuation coefficients was not a particular goal. Results from one blue shark block, spanning the outer edge of the cone wall to the intermedialia, are presented here.

Energy dispersive x-ray diffraction mapping of a blue shark centrum in 50\% ethanol was performed at beamline 6-BM-B, APS, and employed a unique array of ten energy-sensitive detectors. ${ }^{13}$ Figure 2 shows a schematic of instrument and the three orthogonal axes $X$ (horizontal, perpendicular to the incident beam), $Y$ (vertical, normal to the incident beam), and $Z$ (horizontal, parallel to the incident beam). A collimator forms a pencil beam of polychromatic

(a)

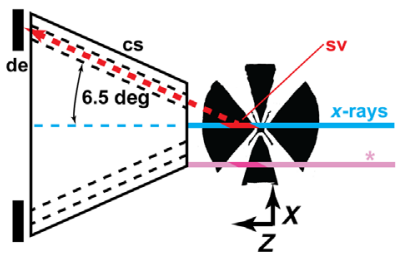

(b)

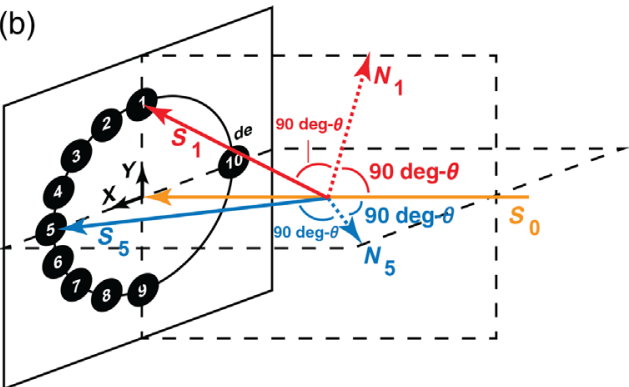

Fig. 2 (a) Schematic of the EDD with a transverse section of the blue shark centrum. Labels: sv, sampling volume; cs, conical receiving slits; de, detector elements. (b) Detector array with detector 1-10 indicated. The detector pair 1 and 9 are at azimuths 180 deg apart as are the pair 5 and 10; detectors 1-9 are equally spaced in azimuth (22.5-deg apart). The distance (along Z) between the sampling volume and the front of the detector slits is $\sim 135 \mathrm{~mm}$, and this represents the maximum sample dimension along this axis that can be scanned without remounting the specimen. Arrangement of detector elements $1-10$ and the orientation of different lattice plane normals $\mathbf{N}_{1}$ and $\mathbf{N}_{5}$ for the crystallites producing diffracted intensities measured in detectors 1 and 5 . 
radiation (dimensions $\delta X \times \delta Y$ of $0.1 \times 0.2 \mathrm{~mm}$ ) which passes through the specimen (here, a transverse section near the middle of a blue shark centrum). Conical receiving slits "cs" (circular slits at two planes with precisely measured separation) block all radiation except that diffracted from the sampling volume "sv" at an angle $2 \theta=6.5 \mathrm{deg}$. By design, the sampling volume is $136 \mathrm{~mm}$ away from front of the conical slits, and assuming adequate $\mathrm{x}$-ray transmission, one could scan specimens up to $\sim 130 \mathrm{~mm}$ in dimension along the beam direction $\mathrm{Z}$. Beamline 6-BM-B normally operates with a large sample compression apparatus in place, and the present experiments were performed with the press in place which, for significant $Y$ movements, limits $Z$ translations to $\sim 80 \mathrm{~mm}$.

The incident beam gage length was $\delta Z=1.7 \mathrm{~mm}$, as determined by translating a $\sim 0.9 \mathrm{~mm}$ thick powdered ceria standard (NIST SRM 674B) across the sampling volume and by measuring the full-width at half-maximum of diffracted peak intensities. The centrum was scanned across the sampling volume using translation increments of $\Delta X=2.0 \mathrm{~mm}, \Delta Y=1.0 \mathrm{~mm}, \mathrm{~s}$ and $\Delta Z=$ $1.9 \mathrm{~mm}$ and $30 \mathrm{~s} /$ position exposures. The integrated intensity for each reflection was determined by fitting with a pseudoVoigt function. ${ }^{6,14}$ The $3 \mathrm{D}$ volume was reconstructed on a $0.5 \mathrm{~mm} \times$ $0.5 \mathrm{~mm} \times 0.5 \mathrm{~mm}$ grid with interpolation of the neighboring voxels and smoothing. The ten energy-sensitive detectors [Fig. 2(b)] simultaneously collect diffracted intensity from crystallites with different orientations.

Shark centra contain a myriad of nanoparticles of bioapatite, ${ }^{6}$ and some of them are oriented to diffract one periodicity ( $d$-spacing $d_{A}$ ) selecting x-ray photons with energy $E_{A}$ and others another periodicity $d_{B}$ with energy $E_{B}$. Each detector records intensity as a function of x-ray energy (see Supplemental Material), and different periodicities produce diffraction peaks at different energies. From the known energy (and its inverse, x-ray wavelength $\lambda$ ), Bragg's law, $\lambda=$ $2 d_{\mathrm{hkl}} \sin \theta$, allows values of $d_{\mathrm{hkl}}$ to be determined for each peak, given the diffraction angle is $2 \theta=6.5 \mathrm{deg}$. The analysis presented below focuses on the integrated intensity of the 00.2 peak, the unresolved 21.1, 11.2, 13.0, and 20.2 quadruplet of peaks hereafter designated peak "q" and the 13.0 peak. The energies of the diffracted $\mathrm{x}$-rays were 32,40 , and $48 \mathrm{keV}$, respectively. It is noteworthy that the use of the abbreviated Miller-Bravais indexing system ${ }^{15}$ that emphasizes the hexagonal crystal system of the bioapatite.

The orientation of the crystals diffracting into detector 5 is given by orientation of lattice plane normal $\mathbf{N}_{5}$ which makes an angle $(90-\theta)$ deg from both the incident beam $\mathbf{S}_{0}$ and the diffracted beam direction $\mathbf{S}_{5}$ and which lies in the horizontal plane [Fig. 2(b)]. The same is true for each other detector, i.e., for detector 1, normal $\mathbf{N}_{1}, \mathbf{S}_{0}$ and the diffracted beam direction $\mathbf{S}_{1}$ are coplanar (vertical plane) and make the same angles. Therefore, the comparison of 00.2 diffracted intensities between different detectors gives the relative fractions of crystalline bioapatite with different orientations defined by their lattice normal orientations $\mathbf{N}_{i}$, i.e., the crystallographic texture within the sampling volume sv.

\section{Results and Discussion}

The results and their interpretation are divided into several subsections beginning with lab microCT and then proceeding to $\mathrm{x}$-ray fluorescence mapping, synchrotron microCT, and 3D EDD mapping. The final section synthesizes what has been learned and where future research might answer outstanding questions.

\subsection{Laboratory MicroCT}

Figure 3 shows lab microCT images of an entire blue shark centrum. The three orthogonal sections reveal the geometry of the cone "c" (corporus calcerea), wedge "W" (intermedialia), and gap " $\mathrm{g}$ " (between mineralized wedges). The cone appears to average higher mineral density than the wedges. In the transverse section, narrow high attenuation features run circumferentially within the wedges; these are growth bands and one is labeled "gb." Numerous other circumferential structures appear between the periodic high attenuation bands. The growth bands are also visible in the two axial sections. Cartilage canals can barely be seen running radially within the wedges. The area with the box is enlarged in the inset (lower right in Fig. 3) and focuses on a 

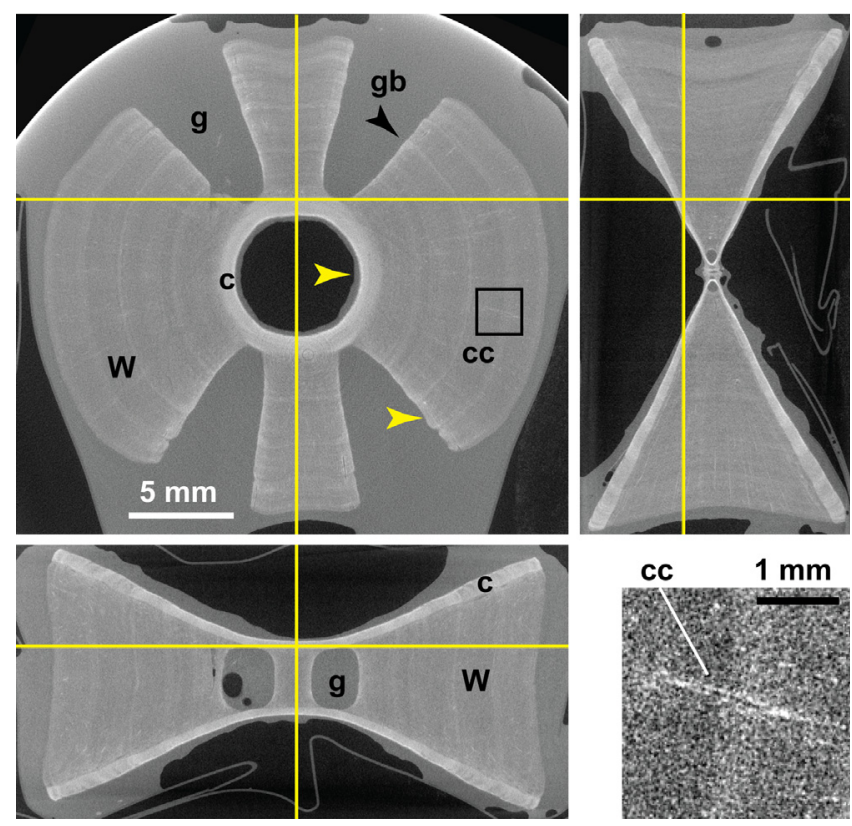

Fig. 3 Three orthogonal sections through the lab microCT data set for the blue shark centrum. The yellow lines indicate the positions of the sections, and the boxed area labeled "cc" is enlarged in the inset panel at the lower right. Here and in Figs. 5 and 6, a linear gray scale is used with the lighter the pixel, the higher the linear attenuation coefficient of the voxel. The horizontal arrowheads point to regions of higher mineral level. The 5- $\mathrm{mm}$ scale bar applies to the three orthogonal sections. The following structures are labeled: W, wedge; c, cone; g, gap between wedges; gb, growth bands; cc, cartilage canal.

single cartilage canal "cc" which is on the order of 2 voxels wide (35 to $40 \mu \mathrm{m}$ ) and which has a border of higher attenuation voxels which one would interpret as a thin hypermineralized zone. Elsewhere $\sim 90-\mu \mathrm{m}$ diameter cartilage canals were seen. The cone wall's external borders and the surfaces of the wedges with the gaps also appear to be hypermineralized.

\subsection{X-Ray Excited X-Ray Fluorescence Mapping}

Figure 4 shows $\mathrm{P}, \mathrm{Ca}$, and $\mathrm{Zn}$ scanning fluorescence maps (blue, red, and green, respectively) of a blue shark centrum section in the orientation indicated in Fig. 1, i.e., at about the same position as the bottom left panel of Fig. 3. The apparent distortion top to bottom of these images is due to the section being slightly oblique relative to the centrum axis. The magenta contrast in the composite $(\mathrm{Ca}+\mathrm{Zn}+\mathrm{P})$ maps shows where $\mathrm{Ca}$ and $\mathrm{P}$ but not $\mathrm{Zn}$ are concentrated, and white contrast indicates high intensities of all three. The cones have higher $\mathrm{Ca}$ and $\mathrm{P}$ intensities than the wedge tissue, and this demonstrates the cones' mineral density is higher than that of the intermedialia. Bands of strong versus low $\mathrm{Ca}$ and $\mathrm{P}$ intensity indicate modulated bioapatite content, and the complex band structure seen in lab microCT is also clear in the $\mathrm{Ca}$ and $\mathrm{P}$ fluorescence maps. The growth band substructure appears less distinct in the $\mathrm{Zn}$ maps, however, perhaps because the $\mathrm{Zn}$ photons emerge from much greater depths than the $\mathrm{Ca}$ or $\mathrm{P}$ signals and slight tilts would obscure narrow features. Such bands were observed previously in X-ray fluorescence maps. ${ }^{16}$ At the right (lateral) side of centrum map in Fig. 4(a), there are a closely spaced series of high intensity $\mathrm{Ca}$ and $\mathrm{P}$ bands, bands which were not seen in the centrum of Fig. 3. The outermost band in the composite $(\mathrm{Ca}+\mathrm{Zn}+\mathrm{P})$ map of Fig. 4(a) is white, indicating that all three elements are concentrated in the lateral outer surface of the intermedialia. A green band is present outside the white band, a characteristic of ongoing biomineralization. ${ }^{17}$ This enhanced $\mathrm{Zn}$ content is, however, non-specific because there are many matrix metalloproteinases and other $\mathrm{Zn}$ containing macromolecules involved in biomineralization. Histology with labels for specific macromolecules would be required to identify the macromolecules present; however, having a specific indication of where to look is very important. The maps of Fig. 4(b) are tilted relative 
(a)
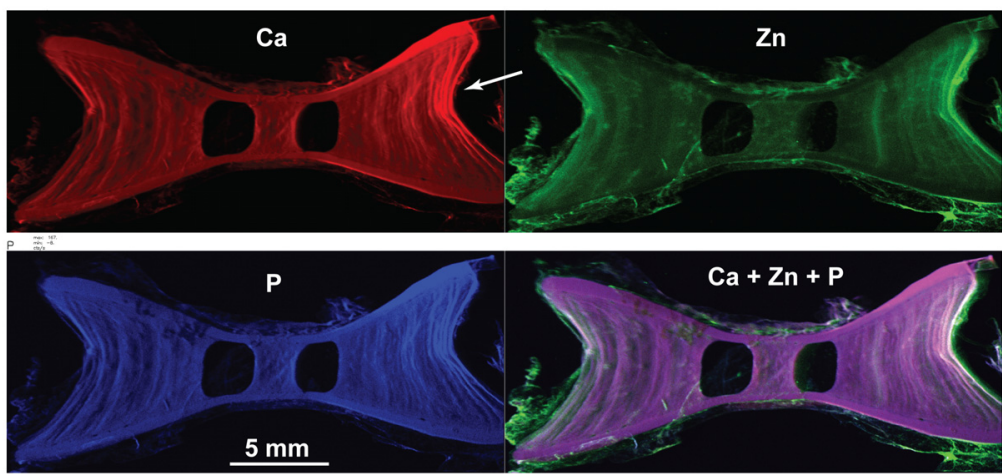

(b)
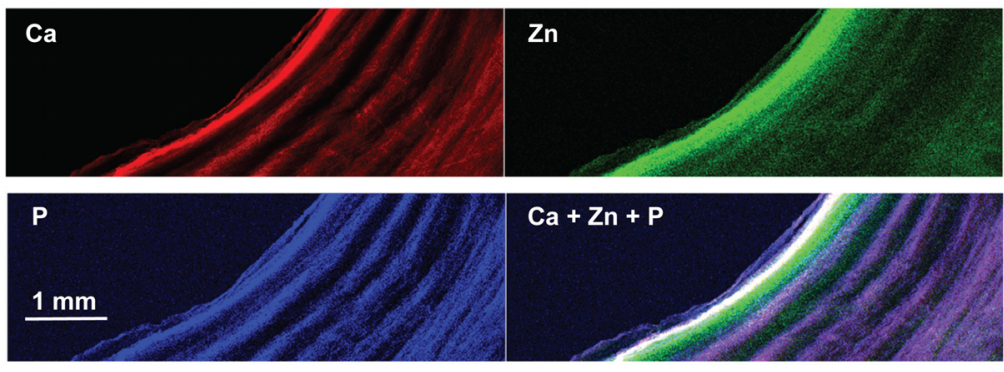

Fig. 4 X-ray excited scanning fluorescence map of $\mathrm{Ca}, \mathrm{Zn}$, and $\mathrm{P}$ intensities along with a composite $\mathrm{Ca}+\mathrm{Zn}+\mathrm{P}$ of the three intensities. Each color scale is linear, extending from zero to that element's maximum, and the brighter the color, the greater the intensity. (a) Map recorded at 8-BM and covering the entire section. The $\mathrm{Ca}, \mathrm{Zn}$, and $\mathrm{P}$ maximum intensities were $7063 ; 176$ and $167 \mathrm{cts} / \mathrm{s}$, respectively. The white arrow in the Ca panel indicates the approximate position mapped at 2-ID-E. (b) Map recorded of a portion of the same section at 2-ID-E. The section was oriented slightly differently than in the 8-BM data, and the horizontal axis of the maps are reflected relative to the first panels. The $\mathrm{Ca}, \mathrm{Zn}$, and $\mathrm{P}$ maximum intensities were $87,324,2223$, and 1516 cts, respectively.

to those in Fig. 4(a) and are reflected from left to right. In Fig. 4(b), the five closely and evenly spaced $\mathrm{Ca}+\mathrm{P}$ bands are present at the outer surface of the wedge. In contrast with Fig. 4(a), the band of increased $\mathrm{Zn}$ content appears inside the increased $\mathrm{Ca}+\mathrm{P}+\mathrm{Zn}$ intensity band, i.e., the green band inside the white band. Note that the earlier study on $\mathrm{Zn}$ bands within shark centra ${ }^{16}$ did not report $\mathrm{Ca}$ and $\mathrm{P}$.

The radial boundaries of the intermedialia are concave in the fluorescence maps of the sections parallel to the vertebral column's axis, something which is also seen, albeit less distinctly, in the upper right numerical section in Fig. 3. The growth bands show this concavity throughout the intermedialia, this indicates that the growing intermedialia's external boundary keeps the same shape throughout. The significance of cone wall growth "leading" intermedialia growth is not clear at present.

\subsection{Synchrotron MicroCT}

Figure 5(a) shows a synchrotron microCT slice from a block cut from the same centrum as the section in Fig. 4. The block is somewhat larger than the FOV (local tomography), so only the image within the cyan dotted circle is accurate. The straight boundary at the bottom of the reconstructed solid is one of the cut surfaces. A more open structure comprises the wedge material "W" which lies to the left of the yellow dotted line segments, and the cone material "c" contains less pore volume (right side of the block). The natural surface of the cone with the intervertebral capsule, labeled " $h$," is the right border of the block, and has the lowest porosity.

Figure 5(b) shows histograms of the cone material and the wedge material [within the boxes in Fig. 5(a)]. The histograms show that the linear attenuation coefficients of the solid portions of cone and wedge are comparable, but the heights of the peaks just below $0 \mathrm{~cm}^{-1}$ in Fig. 5(b) demonstrate quantitatively that fraction of pore voxels is considerably larger within the wedge 


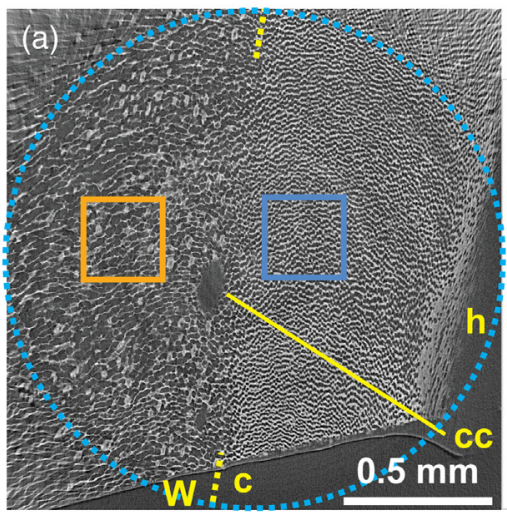

(b)

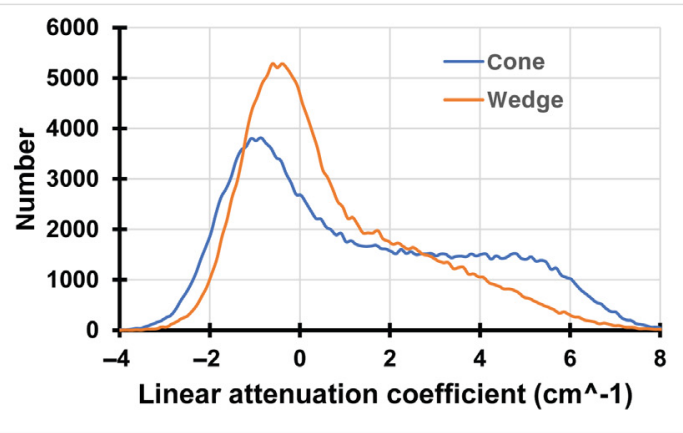

Fig. 5 (a) Synchrotron microCT slice of the blue shark centrum block with the boundary between cone " $\mathrm{c}$ " and wedge "W" indicated by the yellow dotted line segments. The hypermineralized portion of the cone "h," a cartilage canal "cc" and the two boxes where the histograms were measured are indicated. Reconstruction of the volume within the cyan dotted circle is with $0.69 \mu \mathrm{m}$ voxels. (b) Histograms of $400 \times 400$ voxel portions of the cone (blue) and wedge (orange) materials.

than in the cone. The differences in contrast between cone and wedge material seen in lab microCT (Fig. 3) and reported elsewhere for three carcharhiniform sharks, ${ }^{7}$ therefore, are due to different volume fractions of porosity.

There are significantly more negative (non-physical) values in this histogram than are typically seen in reconstructions, and this is a well-known consequence of local tomography with a significant amount of mass outside the FOV, e.g., Stock. ${ }^{12}$ Here, synchrotron microCT is used to explain contrast in the lab microCT data, to identify micrometer-scale structures for further higher resolution, higher contrast sensitivity investigation. Interpretation of the contrast of the mineralized voxels is beyond the scope of this paper.

A cartilage canal "cc," cut obliquely by the slices, is present in the wedge material near the cone-wedge interface [Fig. 5(a)]. The canal diameter is about $80 \mu \mathrm{m}$, and the canal's axis is the radial direction of the centrum. In the lab microCT reconstruction with $18.4-\mu \mathrm{m}$ voxels, one can barely resolve cartilage canals in the blue shark wedges.

Figure 6 enlarges a $400 \times 400 \times 201$ voxel volume surrounding cartilage canal "cc" of Fig. 5 and shows three orthogonal sections through this volume. The transverse section (upper left of Fig. 6) is in the same orientation as the slice in Fig. 5, the three yellow arrowheads labeled " $i-i i i$ " indicate the positions of the sections to the right of the transverse section, and the yellow arrowhead labeled " $i v$ " shows the position of the orthogonal section below the transverse image. The horizontal green arrowheads indicate small 15 to $20 \mu \mathrm{m}$ diameter areas enclosed by a thin layer of mineral; sometimes these appear to contain mineralized material. The side view section ("ii" of Fig. 6) shows that the cartilage canal "cc" runs obliquely through the volume. Side view section " $i$ " demonstrates that the enclosed areas noted in the transverse section are micro-rods aligned parallel with the cartilage canal and consisting of a heavily mineralized periphery and a less mineralized interior. These micro-rods extend hundreds of micrometers.

\subsection{Three-Dimensional Energy Dispersive Diffraction Mapping}

Figure 7 shows the set of EDD reconstructed sections mapped with the integrated intensity of reflection q (unresolved 21.1, 11.2, 13.0, and 20.2 quadruplet of bioapatite peaks) measured with detector 5. Detector 5 measures intensity from bioapatite crystals oriented with either 21.1, 11.2, 13.0 , or 20.2 lattice planes oriented to diffract in the horizontal plane, that is normal to the vertebral column axis (the centrum axial direction) and along the centrum's radial direction. Reflection q is the strongest in the EDD pattern [Fig. 8(a), Supplemental Material], as expected from the reference patterns of polycrystalline hAP, ${ }^{18}$ and this means the sections shown in Fig. 7 are the most accurate EDD map of the centrum. The cone "c" is labeled in Sec. 1, and its inner diameter decreases until it can no longer be resolved in Secs. 7-11 and then increases from Sec. 12 through 17. Part of the cone (upper boundary of Fig. 7) was not covered due to limited 

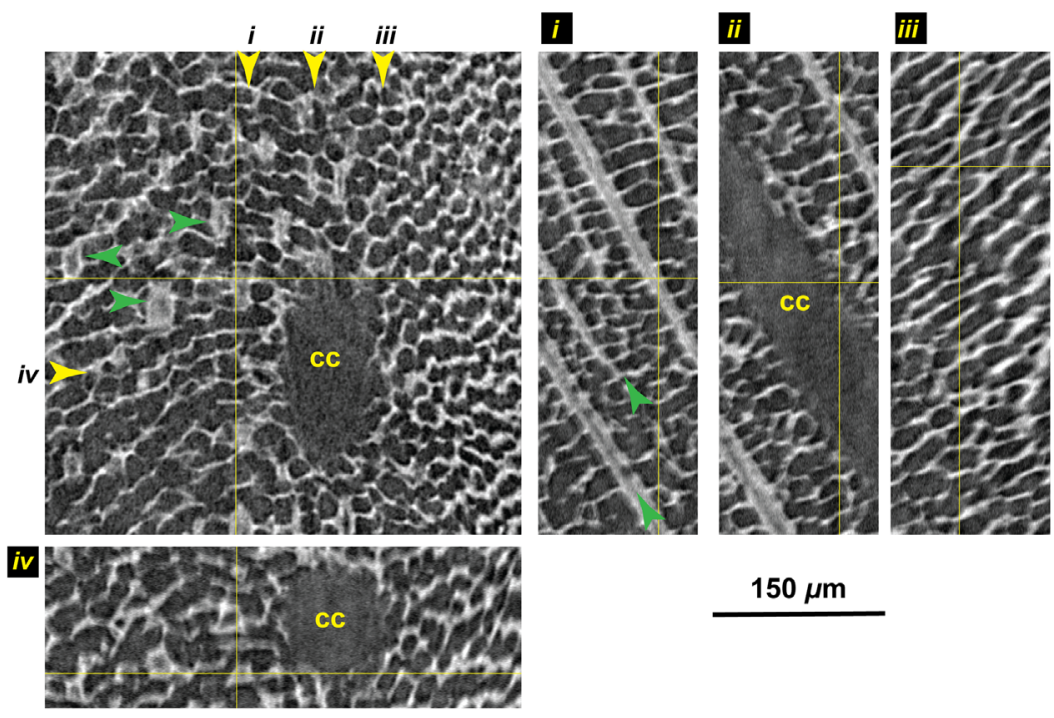

$150 \mu \mathrm{m}$

Fig. 6 Enlargement of the volume around cartilage canal "cc" in Fig. 5. The square images (upper left) is the transverse plane in the synchrotron microCT data set; the position of the orthogonal (axial) slices are indicated by the yellow arrowheads and numerals " $i-i v$." The green arrowheads indicate the calcified micro-rods discussed in the text.

beam time. The wedges begin to be visible in Sec. 3 and are no longer distinguishable in Sec. 15 and after. By Sec. 5 and up to Sec. 12, the wedges and the gaps between them are easily distinguished.

In Fig. 7, very strong quadruplet intensity exists outside the centrum in the lower-left corner of Secs. 5-7 and 12-16 (arrow pointing to Sec. 12). Contrast of each of these two separate features is confined to a few voxels in the plane but extends through multiple sections. The contrast is also seen in precisely the same locations within EDD reconstructions with 00.2 and 13.0 diffracted intensities. Inspection of the lab microCT data set reveals small volumes of mineralized tissue within the neural arch, away from the centrum and precisely where EDD reveals
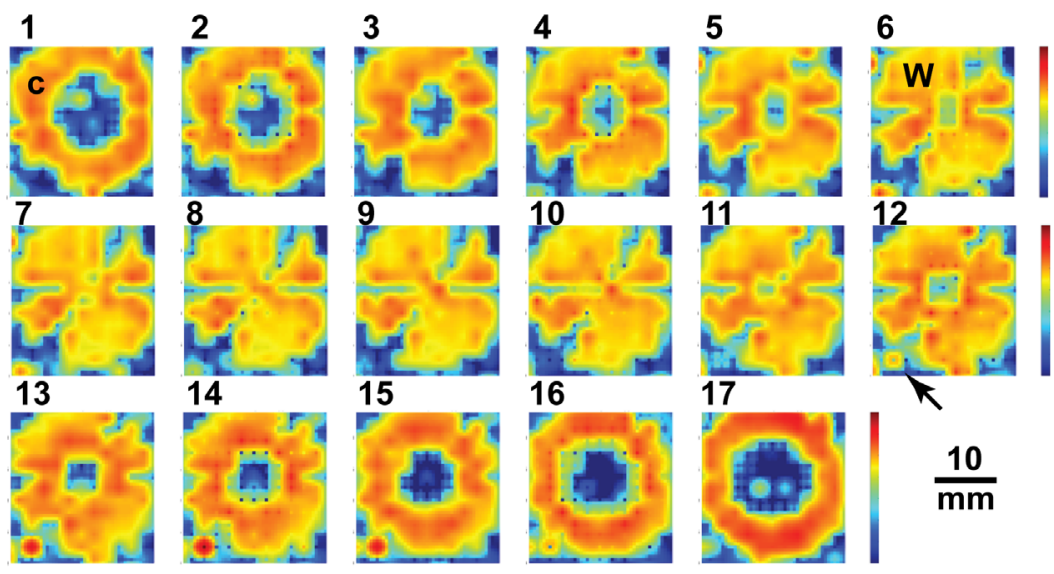

Fig. 7 Series of seventeen contiguous transverse EDD sections reconstructed with intensity from the bioapatite q reflection (unresolved 21.1, 11.2, 13.0, and 20.2 peaks). The section numbers appear above the left corner of each image. The color bars at the right of each row indicate the intensity range (log scale) which was the same for each section. The labels "c" (Sec. 1) and "W" (Sec. 6) identify a cone and wedge, respectively. The arrow (Sec. 12) points to a feature discussed in the text. Ignoring rotation and allowing for the limited spatial resolution, Secs. 6 and 12 agree well with the lab microCT transverse section in Fig. 3 . The variation of diffracted intensity within the wedges in Secs. 6-12 probably represents noise in the data and effects of the interpolation procedure. 
(a)

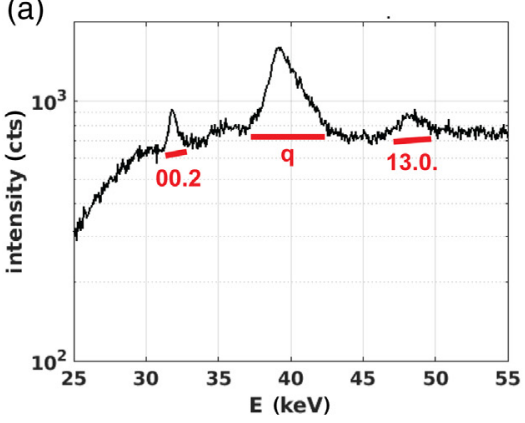

(b)

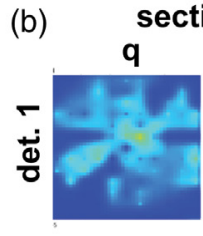

section 9

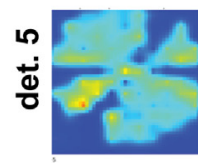

section 16
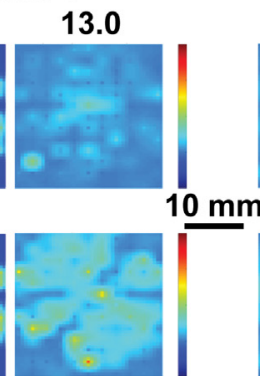

13.0

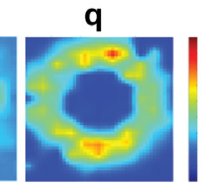

$0 \mathrm{~mm}$

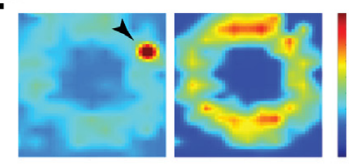

Fig. 8 (a) EDD pattern for the blue shark (sum of patterns for one $Z$ axis scan through the centrum). The 00.2, quadruplet "q" and 13 reflections are labeled. (b) Detector 1 and 5 reconstructions with the quadruplet and 13.0 intensities. Section 9 (wedges of the intermedialia) and 16 (cone, corporus calcerea) are shown. The color bars indicate the linear intensity scale which has a minimum of -50 units and a maximum of 150 for 13.0 and of 400 for the quadruplet. The spike of intensity in the det 5 , Sec. 16, 13.0 reconstruction (black arrowhead) is discussed in the text.

diffracted intensity. Such patches normally occur within shark neural arches. ${ }^{19,20}$ It is noteworthy that almost all of the neural arch was cropped from the image shown in Fig. 3, mainly to allow the features within the centrum to be seen more clearly.

Each detector of the 6-BM-B array samples differently oriented crystallites, and comparison of different detector maps for a given reflection reveals the presence or absence of crystallographic texture. X-ray diffraction (and small-angle scattering) of blocks cut from shark centra showed large azimuthal variation of 00.2 diffracted intensity, i.e., significant $c$ axis texture along well-defined anatomical directions. ${ }^{6}$ Analogous preferred orientation of bioapatite $c$ axes exists in other collagen-based mineralized tissues including bone, ${ }^{14}$ dentin, ${ }^{21}$ and cementum. ${ }^{17}$ Although the individual diffraction peaks within the unresolved quadruplet reflection exhibit crystallographic texture, as can be seen in enamel, ${ }^{22}$ the very broad diffraction 21.1, 11.2, 13.0, and 20.2 peaks (from very small crystallites) of bone, dentin, and cementum produce a combined peak which does not exhibit strong texture. In addition to the relatively strong 00.2 and quadruplet peaks, Fig. 8(a) shows very weak but measurable 13.0 intensity in the EDD patterns from the blue shark centrum. Reconstruction with 13.0 diffracted intensity, therefore, represents a stringent test of sensitivity limits for EDD mapping. In addition, reconstruction with 13.0 intensity could provide additional texture information because the 13.0 reflection is from an $a-b$ lattice plane and is normal to the $c$ axis.

Figure 8(b) compares EDD intensity maps of two sections (9 and 16) for two orthogonally oriented detectors (1 and 5) and for two reflections (q and 13.0). Section 9 covers the wedges of the intermedialia near the centrum's axial midplane, and Sec. 16 intersects one cone near its fullest diameter. The quadruplet maps clearly show the geometry of the structure. In Sec. 16 of the cone, little difference exists between detector 1 and 5 maps of quadruplet intensity; and, in Sec. 9, the quadruplet intensity maps match except for a slight difference in the bottom-most wedge intensity. For the 13.0 reflection, the detector 1 and 5 reconstructions of the cone do not appear to differ from each other nor from those of the other eight detectors (data not shown). This means that, within the cone, 13.0 does not have a preferred orientation. In the 13.0 reconstruction of Sec. 9, however, the wedges cannot be made out in the detector 1 reconstruction but can be seen clearly in that of detector 5 . Therefore, the 13.0 normals within the wedges tend to lie laterally, i.e., perpendicularly to the centrum axis, and not along the centrum's axis. Figure 9 summarizes the EDD observations of bioapatite texture.

Within the 13.0 maps of Fig. 8(b), the intensity is very low, and there are "spikes" of intensity at various positions. The authors inspected each of these positions and confirmed that the automated peak fitting was problematic at these positions. This result indicates a practical limit for EDD reconstruction with weak reflections. It may be that increasing the counting time by a factor of four (and improving the signal to noise ratio by a factor of two) would help to "stabilize" the 13.0 reconstruction, but verifying this requires additional work. On balance, 


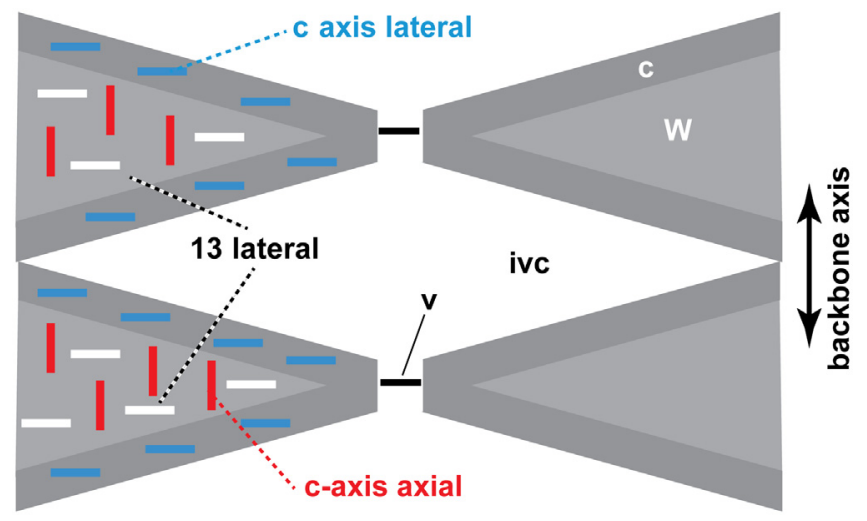

Fig. 9 Summary of crystallographic texture in the blue shark centrum observed by EDD. The red and cyan bars indicate the $c$ axis orientations determined previously. ${ }^{23}$ The white bars indicate the 13.0 preferred orientation in the wedges (but not in the cones). Labels: c, cone; W, wedge; ivc, intervertebral capsule; v, vellum separating adjacent ivc.

however, the 13.0 reflection maps of the blue shark centrum add relatively little to what is determined via the 00.2 and quadruplet maps.

As mentioned above, the quadruplet reflection's insensitivity to texture is expected. The earlier report ${ }^{23}$ on EDD from the same blue shark centrum, showed that the bioapatite $c$ axes were aligned laterally (i.e., perpendicular to the centrum's axis) within the cone and axially within the wedges of the intermedialia (Fig. 9). Given that the 13.0 diffraction planes are perpendicular to the 00.2 planes, the present observation of 13.0 oriented laterally in the intermedialia is consistent with the 00.2 observation. For the cone section in Fig. 8(b), little difference is seen between the two detector maps and this is surprising, at first glance, given the strong 00.2 texture. However, if the bioapatite in the cones has fiber texture (that is, only one direction is strongly oriented ${ }^{15}$ ), 13.0 has rotational freedom around the $c$ axis preferred direction, and 13.0 diffracted intensity would not vary with azimuth.

\subsection{Discussion}

The picture of the blue shark centrum which emerges from x-ray scattering with monochromatic $\mathrm{x}$-radiation (data not presented here), ${ }^{6}$ from synchrotron microCT, from scanning fluorescence microscopy, from lab microCT, and from 3D EDD mapping is one of a hierarchy of structures spanning a wide range of spatial scales, a common theme in bioapatite mineralized tissues. ${ }^{24}$ Synchrotron microCT revealed a honeycomb of heavily mineralized material with wall thicknesses $\sim 1$ to $2 \mu \mathrm{m}$ filled with soft tissue or fluid. Within the wedges of the blue shark centrum, 15 to $20 \mu \mathrm{m}$ diameter micro-rods run parallel to cartilage canals for hundreds of micrometers, and walls of the honeycomb structure link adjacent rods, suggesting that the micro-rods serve a mechanical function. The difference in lab microCT contrast between cone and wedge (Fig. 3) is due to the larger proportion of unmineralized volume in the intermedialia than in the cone (Fig. 5). Earlier x-ray diffraction and small-angle scattering ${ }^{6}$ showed that the average bioapatite properties at the nanoscale (lattice parameter, crystallite size, and crystallographic texture) were similar to but not identical to those of bone, a result which is not surprising given that bone is based on a type I collagen matrix and that the shark centrum matrix is cartilage (a tissue combining types I and II collagen and proteoglycans). Taken together, the hierarchy of structures may be the reason that studies ${ }^{25}$ have shown shark centra are much more compliant than bone centra.

It is unclear at this point whether the material making up the mineralized honeycomb has elastic properties similar to bone tissue or whether shark tissue is significantly less stiff at the one micrometer level. Data on 3D printed, elastomer-hAp scaffolds suggest that much of the high compliance of honeycomb-like structures during compression arises from elastic deflection of the struts and that the hAp particles do not carry significant loads. ${ }^{26}$ The observed macroscopic high compliance and large strains to failure ${ }^{25}$ may, therefore, be due in part to the open 
micrometer-level structure of the centrum tissue and in part to the macroscopic architecture of the centrum. Whether or not the bioapatite-containing tissue (honeycomb walls) are experiencing large strains could be directly tested via in situ loading and position-resolved x-ray strain measurement (the bioapatite nanoparticles would function as embedded strain gages ${ }^{14,21,25}$ ). Given the dimensions of the blue shark wedges and cones, EDD with the 6-BM-B instrument should be able to measure such strains once a loading rig is devised.

Growth bands seen in the fluorescence maps (Fig. 4) and in lab microCT (Fig. 3) scans are not readily apparent in the synchrotron microCT data sets mainly because the FOV is so small; in line with the difference in pore volume fraction observed between cone and wedge (Fig. 5), the authors speculate that the growth bands reflect small local perturbations in pore volume fraction might be quantifiable with considerable effort. The fluorescence maps of the growth bands, in particular, the intensity of $\mathrm{Zn}$ provides information important to understanding growth of the centra, but this is not the focus of the present discussion.

The centrum geometry measured from lab microCT could be imported into 3D models and the structural response could be calculated numerically based upon realistic boundary conditions including the presence of the fluid-filled intervertebral capsule. The EDD maps show strong crystallographic texture at the $0.5-$ to $1-\mathrm{mm}$ scale, and this would require modelers to adjust elastic constants for the cone wall vs. the wedge in order to capture the true response.

Additional EDD mapping of shark centra is clearly required to bolster the conclusions of this study. Perhaps finer scale maps over smaller portions of each centra would reveal nuances not noted here. Centra from additional species and from other carcharhiniform families should be mapped, with particular attention paid to 00.2 texture in the cone vs the wedges. It would also be interesting to extend the mapping to sharks of Order Lamniformes, characterized by intermedialia consisting of a set of radial lamellae instead of wedges. With such different microarchitecture and with the observation that mean mineral levels (at the $20-\mu \mathrm{m}$ level) within cone and lamellae do not differ in lamniforms (unlike in the carcharhiniforms), ${ }^{7}$ crystallographic texture may be absent. However, cartilage fiber orientations within cone and intermedialia, and hence bioapatite crystallographic texture, are likely basal characteristics of sharks and probably do not differ between the orders.

The EDD map of the blue shark centrum was one of the first obtained by the authors; as such, the experiment was aimed primarily at covering the entire centrum and establishing where scanning improvements were needed. Even in this first study, the spatial variation of bioapatite texture and its relationship to anatomical structures was uncovered. Given the similarities of centra across Order Carcharhiniformes, ${ }^{7}$ the authors predict that the lateral orientation of $c$ axis texture in the cones and axial $c$ axis texture in the wedges will be present throughout this order, something that will be proved or disproved in future studies.

At present, it is unlikely that EDD will be able to reconstruct shark centra on 3D grids smaller than $0.5 \mathrm{~mm}$. Relatively low diffracted intensities and long counting times coupled with finite beam time allocation are a significant constraint. Second, the sampling volume dimension along $Z, \delta Z=1.7 \mathrm{~mm}$ here, remains a major limitation. Oversampling along the $Z$ axis direction, e.g. using $\Delta Z=0.5 \mathrm{~mm}$ instead of $\Delta Z=1.9 \mathrm{~mm}$, should allow extraction of a finer $Z$ grid spacing. Alternatively, if one needed higher resolution on a single anatomical plane [say, the transverse plane midway between the centrum's caudal and rostral ends, the $X-Z$ plane in Fig. 2(a)], one could scan the centrum using $\Delta Z$ matching the sampling volume depth $(1.7 \mathrm{~mm})$, rotate $90 \mathrm{deg}$ about the $Y$ axis in Fig. 2(a) and repeat the scan; with the sampling volume's long dimension along two different anatomical directions, one should be able to discern sub-voxel features.

Use of $\sim 70-\mathrm{keV}$ monochromatic photons at a beamlines, such as 1-ID, APS, and with sawtooth focusing-optics to define the pencil beam and conical slits ${ }^{27}$ or spiral slits ${ }^{28}$ to define a sampling volume, is an alternative to EDD mapping. Similar $X-Y-Z$ translation would allow 3D reconstruction of the volume, and different azimuths would be collected simultaneously. At APS, the 1-ID insertion device has orders of magnitude greater flux at any selected wavelength compared to 6-BM-B, and the APS upgrade (estimated completion April 2024) will increase this disparity. Pattern collection speed would increase enormously compared to the current EDD setup. Conical slits tailored to the hexagonal apatite crystal system do not appear to exist, limiting the number of diffraction peaks that can be collected simultaneously, but spiral 
slits can capture all of the hexagonal reflections at the same time albeit with azimuthal gaps in the diffraction rings. It is, however, extremely difficult to obtain beam time at 1-ID, APS, so EDD at 6-BM-B, APS, remains an extremely attractive option.

An alternative to $3 \mathrm{D}$ texture mapping via slit-based approaches is $\mathrm{x}$-ray diffraction tomography ${ }^{29}$ with an incident monochromatic pencil beam and open area detector. In diffraction tomography of fine-grained specimens, one translates the specimen across the beam recording a 2D diffraction pattern at each position, rotates the specimen and repeats the translation and diffraction pattern collection until all viewing angles are recorded. Reconstruction of crosssectional variation of diffracted intensity employs back projection or other computed tomography algorithm. Compared to the translation-rotation method, 3D EDD mapping has the advantage of isolating diffraction from the sampling volume from all other scattering. Additionally, EDD mapping does not require specimen rotation, and specimens with complex surrounding tissue can be aligned so these structures do not affect the diffraction signal.

Use of monochromatic high-energy x-rays $(E>70 \mathrm{keV})$ lessens specimen dose compared to polychromatic x-rays. First, photons in the 25 to $55 \mathrm{keV}$ range (the EDD data) deposit much more energy per photon than those at $70 \mathrm{keV}$ and above. Second, in EDD, photons with energies between those diffracted into the detectors, deposit energy in the specimen and do not contribute signal. Because accumulated x-ray damage affects mechanical properties of collagen-based mineralized tissues, ${ }^{30}$ this could become problematic in in situ loading experiments where the same volume is observed repeatedly.

The EDD mapping approach could certainly be applied to clinically significant specimens, either cadaveric human tissue or tissue from large animal translational models. Mapping in 3D with EDD could probe whether bioapatite crystallographic texture varied between pathological bone and adjacent normal bone, e.g., in osteoarthritis or osteoblastic bone tumors. It could also be used to look for differences between native bone and healthy bone grown as a result of healing, e.g., the bone regrown in lumbar spine fusion operations; that is not to say that the bone would not eventually remodel to look more like the bone formed over a lifetime, but the mineral of the healed bone could initially be very different crystallographically. This information could suggest new clinical approaches. In situ loading combined with EDD mapping and strain quantification might also be very valuable in understanding load distributions in complex assemblies of bone. The authors feel it will be very interesting to see how this technique proves out in the coming years: it certainly cannot be applied to every problem or condition, but 3D EDD tomography might provide key information.

\section{Disclosures}

The authors have no conflicts of interest. Supplemental Material included with this work was not peer reviewed, but was assessed for appropriateness and value as supporting information to the submitted manuscript.

\section{Acknowledgments}

The lab microCT data were collected at the Duke University Shared Materials Instrumentation Facility (SMIF), a member of the North Carolina Research Triangle Nanotechnology Network (RTNN), which is supported by the National Science Foundation (Grant No. ECCS-1542015) as part of the National Nanotechnology Coordinated Infrastructure (NNCI); we thank Justin Gladman for his support of this imaging. Beamline 6-BM-B, APS, is supported by COMPRES, the Consortium for Materials Properties Research in Earth Sciences under NSF Cooperative Agreement EAR-1661511. This research used resources of the APS, U.S. Department of Energy (DOE) Office of Science User Facility, operated for the DOE Office of Science by Argonne National Laboratory under Contract No. DE-AC02-06CH11357. Argonne National Laboratory's work was supported by the U.S. Department of Energy, Office of Science, Office of Basic Energy Sciences, under contract DE-AC02-06CH11357. 


\section{References}

1. M. E. Porter and J. H. Long Jr., "Vertebrae in compression: mechanical behavior of arches and centra in the gray smooth-hound shark (Mustelus californicus)," J. Morphol. 271, 366-375 (2010).

2. M. E. Porter, Jr. et al., "Built for speed: strain in the cartilaginous vertebral columns of sharks," Zoology 117, 19-27 (2014).

3. Y. Y. Watanabe et al., "The slowest fish: swim speed and tail-beat frequency of Greenland sharks," J. Exp. Mar. Biol. Ecol. 426/427, 5-11 (2012).

4. M. R. Urist, "Calcium and phosphorus in the blood and skeleton of the Elasmobranchii," Endocrinology 69, 778-801 (1961).

5. M. N. Dean, W.-A. Chiou, and A. P. Summers, "Morphology and ultrastructure of prismatic calcified cartilage," Microsc. Microanal. 11, 1196-1197 (2005).

6. J.-S. Park et al., "Mineral in shark vertebrae studied by wide angle and by small angle x-ray scattering," under preparation (2022).

7. P. E. Morse et al., "Shark vertebral microanatomy and mineral density variation studied with laboratory microcomputed tomography," J. Struct. Biol. 214, 107831 (2022).

8. M. E. Porter and R. H. Ewoldt, "Automatic control: the vertebral column of dogfish sharks behaves as a continuously variable transmission with smoothly shifting functions," J. Exp. Biol. 219, 2908-2919 (2016).

9. S. R. Stock et al., "Tomography with energy dispersive diffraction," Proc. SPIE 10391, 103910A (2017).

10. N. Kujala et al., "Kirkpatrick-Baez mirrors to focus hard x-rays in two dimensions as fabricated, tested and installed at the Advanced Photon Source," J. Synchrotron. Rad. 21, 662 668 (2014).

11. E. Y. Brister et al., "'X-ray fluorescence microscopy: a method of measuring ion concentrations in the ear," Hearing Res. 391, 107948 (2020).

12. S. R. Stock, MicroComputed Tomography: Methodology and Applications, 2nd ed., Taylor and Francis, Boca Raton, FL (2019).

13. D. J. Weidner et al., "Precise stress measurements with white synchrotron x-rays," Rev. Sci. Instrum. 81, 013903 (2010).

14. J. D. Almer and S. R. Stock, "Internal strains and stresses measured in cortical bone via high-energy x-ray diffraction," J. Struct. Biol. 152, 14-27 (2005).

15. B. D. Cullity and S. R. Stock, Elements of X-Ray Diffraction, 3rd ed., Prentice-Hall, Upper Saddle River, New Jersey (2001).

16. V. Raoult et al., "Localized zinc distribution in shark vertebrae suggests differential deposition during ontogeny and across vertebral structures," PLoS One 13, e0190927 (2018).

17. S. R. Stock et al., "Cementum structure in Beluga whale teeth," Acta Biomater. 48, 289-299 (2017).

18. "Powder diffraction file 00-86-2101," International Centre for Diffraction Data.

19. C. Bordat, "Etude ultrastructurale de l'os des vertebres du Sélacien Scyliorhinus canicula L," Can. J. Zool. 65, 1435-1444 (1987).

20. M. N. Dean and A. P. Summers, "Mineralized cartilage in the skeleton of chondrichthyan fishes," Zoology 109, 164-168 (2006).

21. J. D. Almer and S. R. Stock, "Loading-related strain gradients spanning the mature bovine dentinoenamel junction (DEJ): quantification using high energy x-ray scattering," J. Biomech. 43, 2294-2300 (2010).

22. R. Free et al., "A method for mapping submicron-scale crystallographic order/disorder applied to human tooth enamel," Powder Diffr. 35, 117-123 (2020).

23. J.-S. Park et al., "Three-dimensional mapping of mineral in intact shark centra with energy dispersive x-ray diffraction," J. Mech. Behav. Biomed. Mater. under review (2021).

24. H. A. Lowenstam and S. Weiner, On Biomineralization, Oxford University Press, Oxford (1989).

25. D. I. Ingle, L. J. Natanson, and M. E. Porter, "Mechanical behavior of shark vertebral centra at biologically relevant strains," J. Exp. Biol. 221, 188318 (2018). 
26. S. R. Stock et al., "In situ loading and x-ray diffraction quantification of strains in hydroxyapatite particles within a 3D printed scaffold," Materialia 18, 101174 (2021).

27. J. S. Park et al., "Quantifying three-dimensional residual stress distributions using spatiallyresolved diffraction measurements and finite element based data reduction," Exp. Mech. 53, 1491-1507 (2013).

28. R. V. Martins, C. Ohms, and K. Decroos, "Full 3D spatially resolved mapping of residual strain in a $316 \mathrm{~L}$ austenitic stainless steel weld specimen," Mater. Sci. Eng. A 527, 4779-4787 (2010).

29. D. Gursoy et al., "Maximum a posteriori estimation of crystallographic phases in x-ray diffraction tomography," Philos. Trans. R. Soc. A 373, 20140392 (2015).

30. H. D. Barth et al., "Characterization of the effects of x-ray irradiation on the hierarchical structure and mechanical properties of human cortical bone," Biomaterials 32, 8892-8904 (2011).

Biographies of the authors are not available. 